\title{
SIMPLE GROUPS CONTAIN MINIMAL SIMPLE GROUPS
}

\author{
Michael J. J. BARRY AND Michael B. WARD
}
Abstract
It is a consequence of the classification of finite simple groups that every non-abelian simple group contains a subgroup which is a minimal simple group.

A minimal simple group is a non-abelian simple group all of whose proper subgroups are solvable [7]. The minimal simple groups were classified by Thompson in [7, Corollary 1]. It follows from the definition that every finite non-solvable group $G$ contains a subgroups $H$ and $K$ with $K \triangleleft H$ and $H / K$ a minimal simple group. When $G$ is simple we show that $K$ can be chosen to be $\{e\}$. More precisely our result is

Theorem 1. If $G$ is a finite non-abelian simple group then $G$ contains a subgroup which is a minimal simple group.

In some ways, the result seems obvious. What is surprising is that it does not appear in print anywhere and that some experts do not believe it at first glance.

Before we give the proof of the theorem we present a lemma which will help us deal with the groups of Lie type. This lemma was pointed out to us by Gary Seitz.

Lemma 1. Let $q$ be a power of a prime. Suppose $G$ has a subgroup $H$ which is isomorphic to either $S L_{3}(q)$ or $P S L_{3}(q)$. Then either $G$ is a minimal simple group or $G$ contains a proper subgroup which is non-abelian simple.

Proof of Lemma 1: If $q=2$ or 3 , then $S L_{3}(q) \cong P S L_{3}(q)$ is minimal simple. In this case if $H=G$, then $G$ is minimal simple; otherwise $G$ properly contains the simple subgroup $H$. 
We may assume $q \geq 4$. If $q$ is a power of $2, S L_{3}(q)$ contains, in an obvious way, a copy $K$ of the simple group $S L_{2}(q)$. Since $K \cap Z\left(S L_{3}(q)\right)=$ $\{I\}$, it follows that $P S L_{3}(q)$ contains a copy of $S L_{2}(q)$ as well. Assume $q$ is odd. Now $\Omega_{3}(q) \leq S L_{3}(q)$ since the determinant of any orthogonal transformation is \pm 1 and so any element of the commutator subgroup has determinant 1. Because $\Omega_{3}(q)$ is a simple group isomorphic to $P S L_{2}(q)$ it follows that $\Omega_{3}(q) \cap Z\left(S L_{3}(q)\right)=\{I\}$. Hence $P S L_{3}(q)$ also contains a copy of $\Omega_{3}(q) \cong P S L_{2}(q)$. This concludes the proof of Lemma 1 .

Proof: We will show that if $G$ is not minimal simple then $G$ contains a proper subgroup which is non-abelian simple. The result then follows by induction on the order of $G$. By the classification of finite simple groups, $G$ is an alternating group, a group of Lie type, or a sporadic group. Now the alternating group on five symbols is minimal simple and is contained in every alternating group of degree greater than five. The sporadic groups and the Tits' group are dealt with in the following table. We use $[\mathbf{1}]$ to find a maximal subgroup containing a simple group.

\begin{tabular}{|c|c|c|c|}
\hline Group & Maximal Subgroup & Group & Maximal Subgroup \\
\hline$M_{11}$ & $L_{2}(11)$ & $M_{12}$ & $M_{11}$ \\
\hline$M_{22}$ & $A_{7}$ & $M_{23}$ & $M_{22}$ \\
\hline$M_{24}$ & $M_{23}$ & $J_{2}$ & $A_{5}$ \\
\hline$S u z$ & $A_{7}$ & $H S$ & $M_{22}$ \\
\hline$M c L$ & $M_{22}$ & $C o_{3}$ & $H S$ \\
\hline$C o_{2}$ & $M c L$ & $C o_{1}$ & $C o_{2}$ \\
\hline$H e$ & $S_{4} \times L_{3}(2)$ & $F i_{22}$ & $S_{10}$ \\
\hline$F i_{23}$ & $S_{12}$ & $F i_{24}^{\prime}$ & $F i_{23}$ \\
\hline$H N$ & $A_{12}$ & $T h$ & $U_{3}(8): 6$ \\
\hline$B$ & $T h$ & $M$ & $S_{3} \times T h$ \\
\hline$J_{1}$ & $L_{2}(11)$ & $O^{\prime} N$ & $J_{1}$ \\
\hline$J_{3}$ & $L_{2}(19)$ & $L y$ & $G_{2}(5)$ \\
\hline$R u$ & $A_{8}$ & $J_{4}$ & $U_{3}(11): 2$ \\
\hline${ }^{2} F_{4}(2)^{\prime}$ & $L_{2}(25)$ & & \\
\hline
\end{tabular}

So we can assume $G$ is simple of Lie type. If $G$ is a Chevalley group then $G$ has an associated root system. If the root system contains a 
subsystem of type $A_{2}$ then $G$ contains a subgroup which is isomorphic to either $S L_{3}(q)$ or $P S L_{3}(q)$ and we are done by Lemma 1. (Note in a root system of type $G_{2}$ the long roots form a subsystem of type $A_{2}$.) This only leaves the Chevalley groups $P S L_{2}(q), q \geq 4$ and $P S p_{4}(q), q \geq 3$. By a theorem of Dickson [2, Hauptsatz 8.27], if $P S L_{2}(q)$ is not minimal simple then it contains a non-abelian simple subgroup isomorphic to either $A_{5}$ or $P S L_{2}(r)$ where $r$ divides $q$. If we choose the short root in a base of type $C_{2}$ then the subgroup of $P S p(4, q)$ generated by the root subgroups corresponding to this short root and its negative is isomorphic to $P S L_{2}(q)$ which is simple if $q \geq 4$. The group $P S p_{4}(3)$ is isomorphic to $\mathrm{PSU}_{4}\left(2^{2}\right)$ which we will show below contains the simple group $P S L_{2}(4)$.

If $G$ is a Steinberg group then $G$ is one of ${ }^{2} A_{n}\left(q^{2}\right), n \geq 2,{ }^{2} D_{n}\left(q^{2}\right)$, $n \geq 4,{ }^{2} E_{6}\left(q^{2}\right)$ or ${ }^{3} D_{4}\left(q^{3}\right)$. Now if we consider two adjacent roots on the Dynkin diagram of $D_{n}$ or $E_{6}$ which are left fixed by the graph automorphism of order two we see that ${ }^{2} D_{n}\left(q^{2}\right)$ and ${ }^{2} E_{6}\left(q^{2}\right)$ contain a proper subgroup isomorphic to either $S L_{3}(q)$ or $P S L_{3}(q)$. One way to deal with ${ }^{3} D_{4}\left(q^{3}\right)$ is to consult the list of its maximal subgroups in [4]. Another way is via the following argument pointed out to us by Gary Seitz. If $\alpha_{2}$ is the root in the Dynkin diagram of type $D_{4}$ fixed by the graph automorphism of order three then $\left\{ \pm \alpha_{2}, \pm \alpha_{1}+\alpha_{2}+\alpha_{3}+\right.$ $\left.\alpha_{4}, \pm \alpha_{1}+2 \alpha_{2}+\alpha_{3}+\alpha_{4}\right\}$ is a root sytem of type $A_{2}$ which is fixed by the graph automorphism. Hence ${ }^{3} D_{4}\left(q^{3}\right)$ contains a proper subgroup which is isomorphic to either $S L_{3}(q)$ or $P S L_{3}(q)$. If $n \geq 5$ then ${ }^{2} A_{n}\left(q^{2}\right)$ contains a proper subgroup isomorphic to either $S L_{3}\left(q^{2}\right)$ or $P S L_{3}\left(q^{2}\right)$. We see this by considering a pair of adjacent roots at one end of the Dynkin diagram of type $A_{n}$ and the adjacent pair at the other end that they are mapped to by the graph automorphism of order 2 .

The Steinberg groups remaining for consideration are ${ }^{2} A_{n}\left(q^{2}\right)$ for $n=2,3$ and 4. First of all assume that $q$ is a power of 2 . For $n=3$ or 4 , it is clear that ${ }^{2} A_{n}\left(q^{2}\right)$ contains a subgroup isomorphic to the simple group $S L_{2}\left(q^{2}\right)$. We see this by looking at the roots at the ends of the Dynkin diagram of type $A_{n}$. Now ${ }^{2} A_{2}\left(2^{2}\right)$ is not simple. So assume $q$ is even with $q \geq 4$ and $n=2$. Now the group generated by the centers of a pair of opposite 2-Sylow subgroups is isomorphic to the simple group $S L_{2}(q)$. Assume now that $q$ is odd. We will use the identification of ${ }^{2} A_{n}\left(q^{2}\right)$ with $P S U_{n+1}\left(q^{2}\right)$. Since $P S U_{3}\left(3^{2}\right)$ contains the simple group $P S L_{2}(7)$ we will assume $q>3$ when $n=3$. We will exhibit an embedding of $\Omega_{n}^{+}(q)$ in $S U_{n}\left(q^{2}\right)$ for $n=3,4,5$. (Much more is true - see [5, p. 142].) One way to see this is to take an orthonormal basis $\left\{w_{1}, w_{2}, \ldots, w_{n}\right\}$ for a non-degenerate $n$-dimensional hermitian space $\mathrm{W}$ over $\mathbf{F}_{q^{2}}$ with hermitian form $B_{W}$. Let $V$ be the span of $\left\{w_{1}, w_{2}, \ldots, w_{n}\right\}$ over $\mathbf{F}_{q}$. The restriction $B_{V}$ of $B_{W}$ to $V$ makes $V$ into a non-degenerate quadratic 
space over $\mathbf{F}_{q}$. Now $V$ has maximal index. This is true when $n$ is 3 or 5 because all odd-dimensional non-degenerate quadratic spaces over $\mathbf{F}_{q}$ have maximal index; when $n=4$ we see that the form $B_{V}$ has discriminant $1\left(\mathbf{F}_{q}^{*}\right)^{2}$ equal to the discriminant of the orthogonal sum of two hyperbolic planes. Now map $\sigma \in \Omega_{n}^{+}(V)$ to the unique $\tau \in S U_{n}(W)$ with $\tau\left(w_{i}\right)=\sigma\left(w_{i}\right)$ for $1 \leq i \leq n$. When $n$ is odd, $\Omega_{n}^{+}(V)$ has trivial center, whereas $Z\left(\Omega_{4}^{+}(q)\right)=\{ \pm I\}$. Because $Z\left(\Omega_{n}^{+}(q)\right) \subseteq Z\left(S U_{n}\left(q^{2}\right)\right)$, it follows that $P S U_{n}\left(q^{2}\right)$ contains properly the simple group $P \Omega_{n}^{+}(q)$.

Finally we consider the groups of Suzuki and Ree. If $G$ is ${ }^{2} F_{4}\left(2^{2 m+1}\right)$ then $G$ contains a proper subgroup isomorphic to the simple group $S L_{2}\left(2^{2 m+1}\right)$. We see this by looking at the roots at the ends of the Dynkin diagram of type $F_{4}$ which are paired. If $G$ is ${ }^{2} B_{2}\left(2^{2 m+1}\right)$, then $G$ is minimal simple if $2 m+1$ is prime; otherwise $G$ properly contains the simple group ${ }^{2} B_{2}\left(2^{p}\right)$ for any prime divisor $p$ of $2 m+1$ [6]. The centralizer of an involution in ${ }^{2} G_{2}\left(3^{2 m+1}\right)$ has the form $\mathbf{Z}_{2} \times P S L_{2}\left(3^{2 m+1}\right)$ $[3]$.

This concludes the proof of the theorem.

Acknowledgement. This work was done while the first author was on sabbatical at the University of Oregon. He thanks the Department of Mathematics at Oregon for its wonderful hospitality, Allegheny College for its generous support during the sabbatical, and Gary Seitz for a number of helpful conversations.

\section{References}

1. J. H. Conway, R. T. Curtis, S. P. Norton, R. A. Parker and R. A. Wilson, "Atlas of finite groups," Clarendon Press, Oxford, 1985.

2. B. Huppert, "Endliche Gruppen I," Springer-Verlag, Berlin, 1967.

3. Z. Janko and J. G. Thompson, On a Class of Finite Simple Groups of Ree, J. Algebra 4 (1966), 274-292.

4. P. B. Kleidman, The maximal subgroups of the Steinberg triality groups ${ }^{3} D_{4}(q)$ and their automorphism groups, J. Algebra 115 (1988), 182-199.

5. P. Kleidman and M. Liebeck, "The Subgroup Structure of the Finite Classical Groups," Cambridge University Press, Cambridge, 1990.

6. M. Suzuki, On a class of doubly transitive groups, Ann. of Math 75 (1962), 105-145. 
7. J. G. Thompson, Nonsolvable groups all of whose local subgroups are solvable: I-VI, Bull. Amer. Math. Soc. 74 (1968), 383-437; Pacific J. Math. 33 (1970), 451-536;, 39 (1971), 483-534;, 48 (1973), 511-592;, 50 (1974), 215-297;, 51 (1974), 573-630.

Michael J. J. Barry:

Department of Mathematics

Allegheny College

Meadville, PA 16335

U.S.A.

e-mail: mbarry@alleg.edu
Michael B. Ward:

Department of Mathematics

Bucknell University

Lewisburg, PA 17837

U.S.A.

e-mail: mward@bucknell.edu

Rebut el 21 de Març de 1996 